\title{
Inductive Linear Displacement Sensor in Active Magnetic Bearing
}

\author{
Sergei Loginov \\ Department of the electric drive and \\ systems of automation \\ Pskov State University \\ Pskov, Russia \\ 1sy1@mail.ru \\ Igor Plokhov \\ Department of the electric drive and \\ systems of automation \\ Pskov State University \\ Pskov, Russia \\ igor_plohov@list.ru
}

\author{
Dmitriy Fedorov \\ Department of the electric drive and \\ systems of automation \\ Pskov State University \\ Pskov, Russia \\ dmitriy-fedorov-2012@mail.ru \\ Andrey Hitrov \\ Department of the electric drive and \\ systems of automation \\ Pskov State University \\ Pskov, Russia \\ khitrov.aa@gmail.com
}

\author{
Igor Savrayev \\ Department of the electric drive and \\ systems of automation \\ Pskov State University \\ Pskov, Russia \\ igor_savraev@mail.ru \\ Alexander Hitrov \\ Department of the electric drive and \\ systems of automation \\ Pskov State University \\ Pskov, Russia \\ khitrov-pscov@mail.ru
}

\begin{abstract}
Active magnetic bearings are increasingly used in various fields of industry. The absence of mechanical contact makes it possible to use them in ultra-highspeed electric drives. The main trend of active magnetic bearings development is the improvement of the control system. The main problem of the control system is the displacement sensor (most of them has low accuracy and large interference). The sensor must have the following properties: simple in realization, high linearity of the characteristic, high sensitivity and noise immunity, high reliability. At the present time there is no sensor that satisfies all these conditions. Most manufacturers use various kinds of filters to get an accurate position signal. This increases the response time of the control system. Thus, problem of designing and modeling the position sensor, considered in the article is topical.
\end{abstract}

Keywords-active magnetic bearings, inductive sensor of situation, measurement of provision of a rotor.

\section{INTRODUCTION}

The Active Magnetic Bearings (AMB) receive the increasing application in various fields of the industry. Lack of mechanical contact allows to use them in ultrahigh-speed electric drives (now it is in great demand) [4]. An integral part of AMB is the measuring converter of linear movements (the situation sensor). The sensor measures the shift of a rotor of rather central situation. The sensor of situation is one of the major elements in the AMB control system, stability of system depends on its accuracy and speed. Sensor parameters in many respects are defined by its design. Now in AMB inductive, vortical current based, capacitor and magnetic sensors [11] are used. Besides, sensor-less systems [12] can be applied. For improvement of the AMB technical parameters (stability, quality of transitional characteristics, speed) there is urgent a problem of development of sensors of a new design with the improved technical characteristics

$[7,8,9]$.

\section{MATHERIALS AND METHODS}

Active magnetic bearings due to lack of mechanical contact between a rotor and the stator except high-speed appendices allow to work in vacuum, in the conditions of radiation, in hostile environment, in superclean technologies and at low and high temperatures at which operation of ordinary bearings is impossible [1], [2]. The principle of action of AMB is shown in fig. 1.

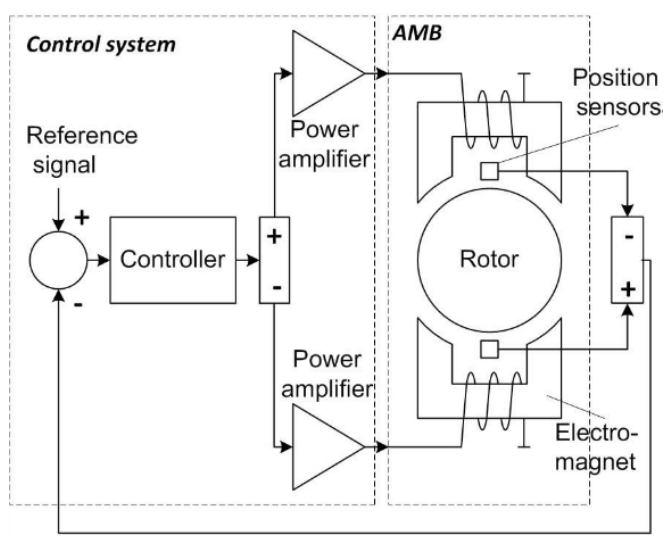

Fig. 1. Principle of action of AMB.

The AMB without control system is unstable system. The correct work is reached only at the expense of feedback by situation and control systems. The provision of a rotor in a gap is defined by situation sensors. By means of a signal of a task it is possible to set micromovements of a rotor in a gap. The controller processes a signal from the sensor on one of laws of management (proportiondifferential, proportional-integral-differential-regulators, adaptive management, an algorithm on the basis of neural networks, etc. $[3,5,6])$. Amplifiers of power set currents in windings of electromagnets. Thus, the electromagnetic forces holding a rotor in a standard position are controlled.

Key element of a control system is the sensor of linear shift of a rotor in radial situation. Properties of all system 
in many respects are defined by its parameters. It has to have high precision of measurements (about several microns), high speed (about several $\mathrm{kHz}$ ), a high noise stability. There is a big variety of sensors of situation, the main can be considered inductive, induction, capacitor and sensors of measurement of magnetic field.

However there is not enough noise stability of any sensor to use it directly for further processing on the controller. For obtaining value of shift of a rotor filters are used (or on active elements, or digital). The problem is that the better filtration requires the big frequency of a signal, otherwise there is an inadmissible delay when processing a signal.

Let's consider the existing inductive sensor of provision of a rotor.

The function chart of the converter (fig. 2) includes the generator of rectangular voltage of the bearing frequency set by the microcontroller, the transformer with two identical windings for obtaining two voltage in an antiphase, the inductive sensor of shift, with two oppositely concerning the center of a rotor the located windings (for receiving a differential signal), the analogdigital converter and the microcontroller which carries out processing of a signal.

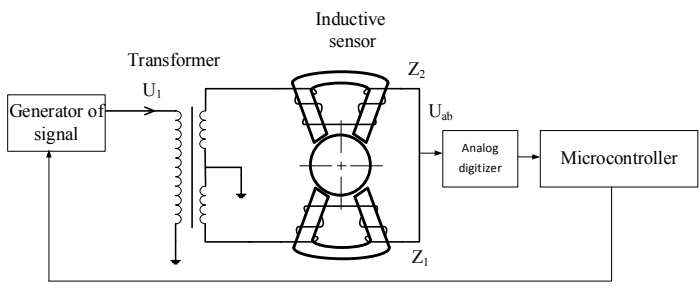

Fig. 2. The function chart of the inductive converter with the transformer bridge.

Secondary windings of the transformer and winding of the sensor form the transformer bridge. In the central provision of a rotor the bridge is balanced also output voltage $\mathrm{U}_{\mathrm{ab}}=0$. In the displaced provision of a rotor we have

$$
U_{a b}=E \frac{Z_{1}-Z_{2}}{Z_{1}+Z_{2}+2 Z}
$$

where E - the electromotive force of a secondary winding of the transformer; - complex resistance of a secondary winding, first and second windings of the sensor respectively.

The bearing frequency is usually high therefore active resistance of windings are negligible in comparison with their inductive resistance. Besides the transformer bridge is designed so that inductance of secondary windings of the transformer was much less inductance of windings of the sensor. Then in the displaced provision of a rotor we have

$$
U_{a b}=\frac{E\left(L_{1}-L_{2}\right)}{L_{1}+L_{2}},
$$

where $L_{1}, L_{2}$ - inductance of windings of the sensor.

The sensor model in the environment of Ansys is presented in the figure 3

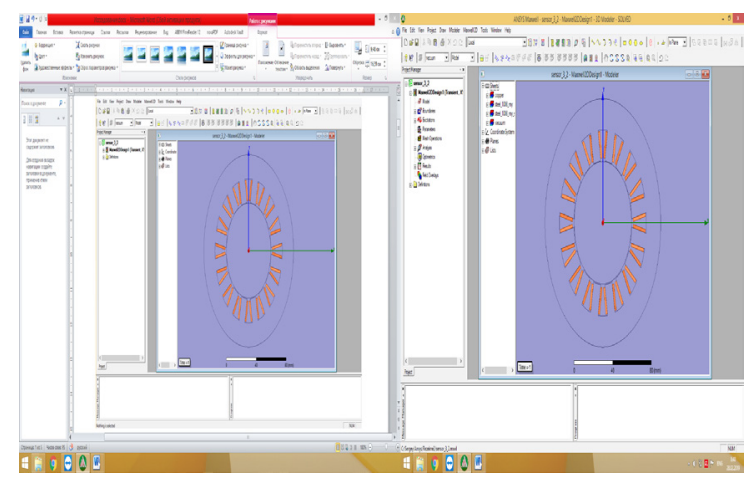

Fig. 3. Shift sensor Model in the environment of Ansys.

The bearing frequency usually is set about $10 \mathrm{kHz}$. It is caused on the one hand by desire to increase speed, on the other hand restriction of speed of analog digitizer of the microcontroller.

When giving on an entrance of basic voltage of squared Uo (fig. 4), at the exit we receive voltage which sign concerning a sign basic will depend on in what party the rotor concerning sensor windings is displaced.

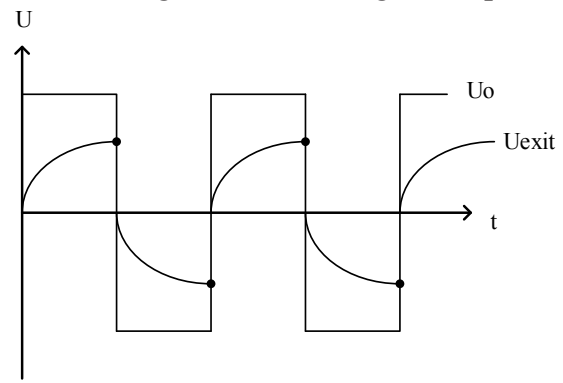

Fig. 4. Basic and output voltage of the sensor of shift.

At low frequencies output voltage has almost rectangular shape. At frequencies about tens $\mathrm{kHz}$ vortex currents, despite execution of a from leafs made magnetic conductor of the sensor have significant effect. Therefore we observe exponential increase of current. At such form of a signal it is required to perform measurement in a point of the highest value of voltage, but it is necessary to finish transformation of an analog signal before switching of the front of a basic signal. Taking into account that in full AMB there are 5 sensors of situation, speed of analog digitizer has to be 2 orders more than the frequency of a basic signal.

In the figure 5 results of modeling in the environment of Ansys Maxwell at rotor shift in the direction of an axis y on $0.1 \mathrm{~mm}$ are presented.

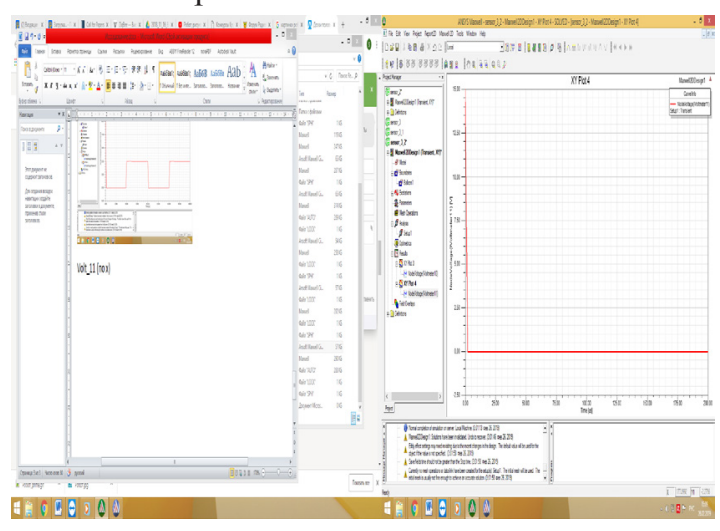

a) 


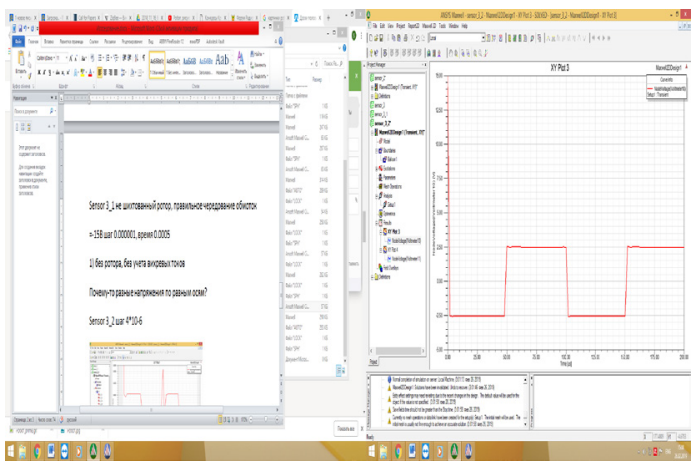

b)

Fig. 5. Results of basic and output modeling in the environment of Ansys Maxwell. a) voltage on axis $\mathrm{X}$ coils) voltage on axis $\mathrm{Y}$ coils.

Initial value of voltage $15 \mathrm{~B}$ is caused by entry conditions when calculating model and does not reflect reality

Now speed of analog digitizer of the microcontroller makes about $1 \mathrm{MHz}$. Thus the frequency of a basic signal is limited to value about $10 \mathrm{kHz}$.

\section{RESULTS AND DISCUSSION}

Application of more high-speed analog digitizer is possible, however for them the range of entrance voltage usually decreases that negatively affects on a sensor noise stability.

In modern microcontrollers there is a module of capture and comparison. It allows to perform measurements of intervals of time between digital signals with a big accuracy and speed. Thus, if to receive a situation signal from the sensor which will be proportional to width of an impulse of a digital signal it is possible to increase speed.

In the figure 6 the function chart of the inductive measuring converter with a digital output is shown.

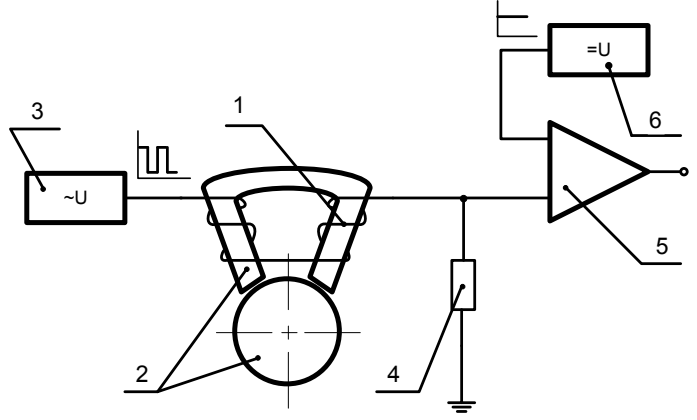

Fig. 6. The functional chart of the upgraded inductive sensor of linear movements.

The inductive measuring converter with a digital output contains the coil of inductance 1 which is put on not closed magnetic conductor with a changeable gap 2 , a source of the rectangular voltage 3 , the resistor 4 , the comparator 5, a source of basic voltage 6 . Consistently to the coil 1 are switched on the generator of rectangular voltage 3 and the resistor 4 , one of conclusions of which is connected to grounding, and another to the coil 1 and one of comparator 5 entrances, the source of basic voltage 6 is connected to other entrance of the comparator 5 .

If in a reciprocal part of a magnetic conductor 2 there are vortex currents, then power failure on the resistor will have the form close to triangular. At change of a gap of the core inductance of the coil and size of vortex currents change, and, therefore, also voltage amplitude on the resistor will change. On entrances of the comparator the constant basic voltage and triangular voltage from the resistor (fig. 7) moves. At the same time at its exit there are impulses which duration the $\mathrm{T}$ depends on magnetic conductor gap size.

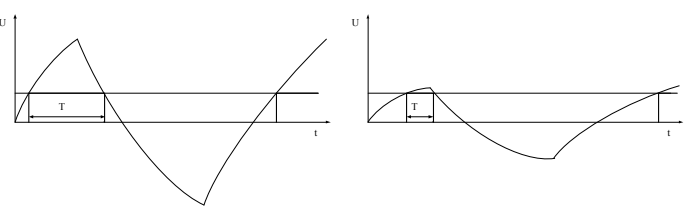

Fig. 7. Formation of impulses on the upgraded sensor. In the figure 8 results of modeling in the environment of Ansys Maxwell with a frequency of $50 \mathrm{kHz}$ are shown.

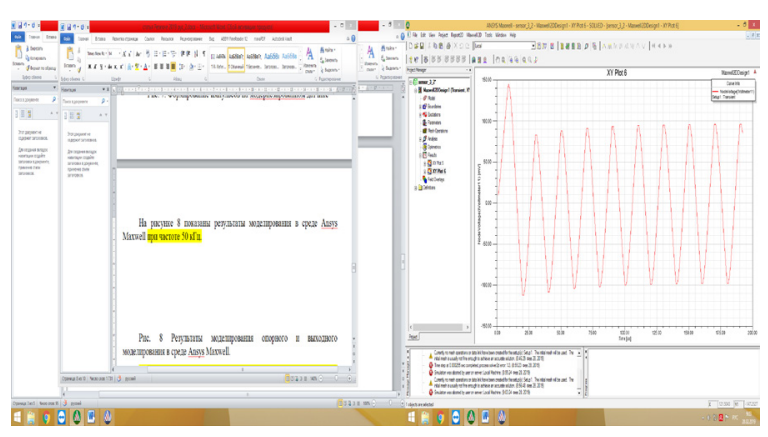

Fig. 8. Results of basic and output modeling in the environment of Ansys Maxwell.

Voltage shift at the initial stage of modeling is caused by entry conditions of calculation. Smaller amplitude value of voltage is caused by the bigger frequency and influence of vortex currents. For increase in sensitivity it is enough to increase sensor supply voltage.

For reduction of losses it is desirable that regarding a magnetic conductor on which the coil is reeled up, there would be no vortex currents, i.e. it needs to be produced from thin sheets of steel or from ferrite.

\section{CONCLUSIONS}

Thus, are presented in article result of computer modeling of the inductive sensor of shift, and the upgraded inductive converter of linear movements used now.

Due to more fast measurement an opportunity to increase the frequency of the signal of basic voltage given on a sensor winding is available the $\mathrm{T}$ time interval microcontroller, than an analog signal. It positively affects on speed of all system and gives the chance of better processing of the received signal digital filters. Besides, there is an opportunity to avoid processing of analog signals that allows to choose more high-speed digital signal processor which is not containing the analog digitizer block. Still, if to place this converter directly with $\mathrm{AMB}$, the hindrances induced at a transmission line of an analog signal from the sensor to the control unit are excluded.

On this inductive converter of linear movements the patent for the invention is taken out [12]. 


\section{ACKNOWLEGMENTS}

This article is prepared with partial support of the Ministry of Education and Science of the Russian Federation. "Development of Bases of the Theory of the Bearingless Synchronous Electrical Machine" project No. $2014 / 700$

This study was partly supported by the Ministry of Education and Science of the Russian Federation. Project "Development of the Bearingless Synchronous Electrical Machine Theory Foundations" No. 2014/700

\section{REFERENCES}

[1] A.Chiba, T.Fykao, O.Ichikawa, M.Oshima, M.Takemoto and D.G.Dorrell, Magnetic Bearings and Bearingsless Drives. ELSEVIER, pp. 127-135.

[2] Y. Zhuravlyov Active Magnetic Bearings: Theort, Design, Aplication. St. Petersburg: Politechnica, 2003, pp. 12-25

[3] Adaptive control of the 1-DOF active magnetic bearing Sergei Loginov, Yuri Zhuravlyov, Yulia Domracheva, Dmitriy Fedorov Environment. Technology. Resources. Proceedings of the 11th International Scientific and Practical Conference. - 2017. volume III - p. 188-191. ISSN 1691-5402

[4] G. Schweitzer, H. Bleuler, and A..Traxler, Active Magnetic Bearings. Zurich, VDF Hochschulverlag AG, pp. 31-77 1994

[5] V.S. Polamraju, G.V. Sobhan, K. Nagesh, J. Amarnath and M. Subbarao. "Stabilization of active magnetic bearing system using single neuron PID controller", ARPN Journal of Engineering and Applied Sciences vol. 9, no. 7, july 2014
[6] Ming-Mao Hsu, Seng-Chi Chen, Van-Sum Nguyen and Ta-Hsiang $\mathrm{Hu}$ "Fuzzy and online trained adaptive neural network controller for an AMB system", Journal of Applied Science and Engineering, vol. 18, No. 1, pp. $47-58.2015$

[7] AN AXIAL POSITION SENSOR FOR ACTIVE MAGNETIC BEARINGS Alexei V. Filatov Lawrence A. Hawkins Proceedings of ASME Turbo Expo 2010: Power for Land, Sea and Air GT2010 June 14-18, 2010, Glasgow, UK

[8] A low cost eddy current displacement sensor for active magnetic bearings A dissertation presented to The School of Electrical, Electronic and Computer Engineering North-West University In partial fulfilment of the requirements of the degree Master Ingeneriae in Electrical and Electronic Engineering by Andries May 2008

[9] Resonant inductive displacement sensor for active magnetic bearings AngeloBonfittoa RanGabaib AndreaTonolia Luis MiguelCastellanosa NicolaAmatia Sensors and Actuators A: Physical Volume 287, 1 March 2019, Pages 84-92

[10] The patent for the invention No. 2557373 "The inductive measuring converter with a digital output" It is registered on June 24, 2015. Loginov Sergey Yuryevich, Domracheva Yulia Vyacheslavovna, Fedorov Dmitry Sergeyevich

[11] Position Sensor. Waukesha Magnetic Bearings position sensors provide [Online]. Available: <https://www.waukbearing.com/en/ magnetic-bearing-systems/system-details/position-sensor/>

[12] Sensorless position control of active magnetic bearings based on high frequency signal injection method Jung-Sik Yim, JangHwan Kim, Seung-Ki Sul, Hyeong-Joon Ahn, Dong-Chul Han Conference: Applied Power Electronics Conference and Exposition, 2003. APEC '03. Eighteenth Annual IEEE, Volume: 1 